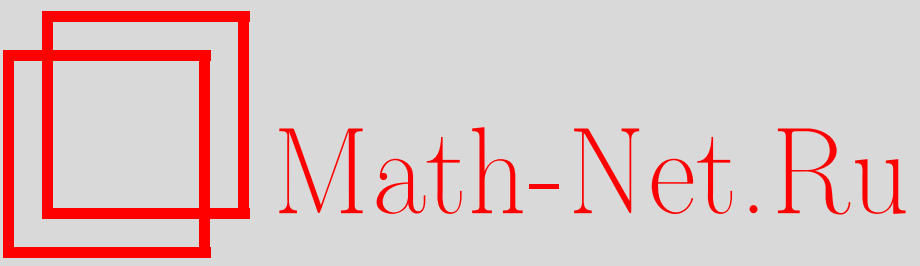

В. Ю. Новокшенов, А. Г. Шагалов, Решения типа связанных состояний для эллиптического уравнения синус-Гордон, ТМФ, 1997, том 111, номер 1, 15-31

DOI: https://doi.org/10.4213/tmf987

Использование Общероссийского математического портала Math-Net.Ru подразумевает, что вы прочитали и согласны с пользовательским соглашением

http://www . mathnet.ru/rus/agreement

Параметры загрузки:

IP : 54.89 .56 .158

26 апреля 2023 г., 10:49:50 
ТЕОРЕТИЧЕСКАЯ

И МАТЕМАТИЧЕСКАЯ

ФИЗИКА

Том 111, № 1

апрель, 1997

В.Ю. Новокшенов* , А.Г. Шагалов

\section{РЕШЕНИЯ ТИПА СВЯЗАННЫХ СОСТОЯНИЙ ДЛЯ ЭЛЛИПТИЧЕСКОГО УРАВНЕНИЯ СИНУС-ГОРДОН}

Детально изучаются решения с конечной энергией и точечными особенностями для эллиптического уравнения синус-Гордон в плоскости. Это решения типа связанного состояния (в смысле скалярной теории поля). Если точка особенности единственна, то на большом расстоянии от нее они представляют собой солитоноподобный кольцевой волновой пакет. Эффективный радиус этого пакета вычислен аналитически и численно для случая осесимметричных решений. Аналитическое исследование основано на методе изомонодромных деформаций для третьего уравнения Пенлеве, которое выделяет эти решения как сепаратрисы многообразия общих решений (с бесконечной энергией). Точные аналитические оценки дают инструмент для изучения решения типа связанных состояний для неинтегрируемого уравнения синус-Гордон с правой частью. Точнее, для полей большой интенсивности в точке особенности мы получаем значение критического возбуждения, допускающее существование и устойчивость связанного состояния. В качестве иллюстрации рассматриваются два приложения - джозефосоновский контакт большой плошади и нематические жидкие кристаллы во врашающемся магнитном поле. Для обоих примеров мы вычисляем критические значения поля, допускающие формирование режимов с конечной энергией. Они хорошо коррелируют с численными и экспериментальными данными.

\section{1. ВВЕДЕНИЕ}

Сингулярные решения эллиптического уравнения синус-Гордон

$$
\varphi_{x x}+\varphi_{y y}=\sin \varphi-f, \quad f=\text { const },
$$

с особенностями вида

$$
\varphi=\alpha \log \frac{1}{r}+\beta+o(1), \quad r^{2}=x^{2}+y^{2} \rightarrow 0,
$$

служат для моделирования точечных дефектов в физике конденсированного состояния. Подобные решения описывают, например, неоднородности сверхпроводящего тока вблизи углов в больших прямоугольных джозефсоновских переходах [1-3], а также поведение директора в нематическом жидком кристалле во вращающемся магнитном поле вблизи частиц примеси [4-7]. Кроме того, уравнение (1.1) следует в некотором приближении [6,

* Институт математики Уфимского научного центра РАН, e-mail: novik@imat.rb.ru

${ }^{\dagger}$ Институт физики металлов, Екатеринбург, e-mail: Arkadi.Shagalov@usu.ru 
8] из анизотропного уравнения Гинзбурга-Ландау и представляет простую модель для фазовых дефектов в этом уравнении.

Другая задача о сингулярных решениях описывает связанные состояния в скалярных теориях поля. Известно, что в классе гладких решений уравнение (1.1) не имеет в $\mathbb{R}^{2}$ связанных состояний $[9,10]$, т.е. решений, обладаюших конечной энергией

$$
E=\int\left[\frac{1}{2} \varphi_{x}^{2}+\frac{1}{2} \varphi_{y}^{2}-\cos (\varphi)+\cos \left(\varphi_{0}\right)-f\left(\varphi-\varphi_{0}\right)\right] d x d y
$$

и доставляюших минимум функционала (1.3), т.е. $\delta^{2} E / \delta \varphi^{2} \geq 0$. На бесконечности поле $\varphi$ выходит на однородное асимптотическое состояние: $\varphi \rightarrow \varphi_{0}, r \rightarrow \infty$. Однако если допустить решения с точечными особенностями (1.2), то глобальные решения с конечной энергией существуют, т.е. связанные состояния становятся возможными. При этом мы должны исключить из области интегрирования в (1.3) маленький диск радиуса $a \ll 1$ вокруг сингулярности, где $a$ - радиус "обрезания", определенный физической применимостью непрерывной модели (1.1) [11].

Обшая структура сингулярных решений была найдена численно в $[2,3,7]$ для уравнения $(1.1)$ и его $(2+1)$-мерной версии

$$
-\varphi_{t}+\varphi_{x x}+\varphi_{y y}=\sin \varphi-f
$$

где уравнение (1.1) описьвает стационарные состояния. Для свободного поля $(f=0)$ такие решения в случае радиальной симметрии сушествуют и ограничены в $\mathbb{R}^{2} /\left\{x^{2}+\right.$ $\left.y^{2}<a^{2}\right\}$, экспоненциально убывая на бесконечности $x^{2}+y^{2} \rightarrow \infty$. На не очень больших расстояниях от центра имеет место промежуточная асимптотика типа радиально-симметричного волнового пакета, которая может интерпретироваться как система кольцевых солитонов уравнения (1.1) с общим центром в сингулярности - структура типа "мишени" $[2-5]$. Вынуждаюшая сила $f$ играет ключевую роль для сушествования решений уравнения (1.1) с конечной энергией. В работах $[3,7]$ было найдено, что для значения $f$, превьшаюшего некоторое критическое значение $f_{c}$, стационарные по $t$ решения становятся неустойчивыми и порождают новые динамические режимы в уравнении (1.4).

Ниже мы подробно исследуем сингулярные решения для радиально-симметричного уравнения (1.1):

$$
\varphi_{r r}+\frac{1}{r} \varphi_{r}=\sin \varphi-f .
$$

Для значения $f=0$ уравнение (1.5) сводится к специальному случаю уравнения Пенлеве третьего типа (РІІІ). Его обшие решения были предметом обширных аналитических исследований методом изомонодромных деформаций [12-14]. Эта техника применима в том числе и для решений типа связанных состояний (см. ниже п. 2.2), которые выступают в качестве одномерного "сепаратрисного" многообразия в двумерном многообразии обших решений уравнения (1.5). Их экспоненциальное убывание на бесконечности

$$
\varphi(r)=2 \pi k+\gamma \sqrt{\frac{\pi}{2 r}} e^{-r}\left(1+O\left(r^{-1}\right)\right), \quad r \rightarrow \infty,
$$


где $k$ - целое число, порождает уравнение на два первых интеграла (данные монодромии), позволяющее явно указать связь констант $\alpha$ и $\beta$ в асимптотике (1.2) в точке сингулярности. Другим следствием этой параметризации данными монодромии являются формулы связи величин $k$ и $\gamma$ в асимптотике (1.6) с начальными данными $\alpha$ и $\beta$.

Далее, из явных формул связи параметров (1.2) и (1.6) мы вычисляем эффективный радиус связанного состояния, который, грубо говоря, есть координата наиболее удаленного кольцевого солитона. Это дает инструмент для изучения решения типа связанного состояния в неинтегрируемом уравнении (1.5) с внешней силой $f$. А именно, для большого $\alpha$ и $|f| \leq 1$ мы получаем критическое значение $f_{c}(\alpha) \approx \mathrm{const} \cdot \alpha^{-1}$, которое контролирует существование и устойчивость решений (1.6) (см. раздел 3 ). В разделе 4 этот эффект иллюстрируется на примере двух конкретных физических приложений - джозефсоновских контактов большой плошади и нематических жидких кристаллов во вращаюшемся магнитном поле. Для обоих примеров мы вычисляем критические значения поля и внешней силы, допускаюшие формирование режимов с конечной энергией. Они имеют хорошую корреляцию с компюютерным моделированием и экспериментальными данными $[3,7]$.

В заключение обратим внимание на связь исследуемых решений с решениями типа связанных состояний для уравнения sinh-Gordon (это уравнение (1.5) с гиперболическим синусом в правой части). Его экспоненциально убывающие на бесконечности решения подробно изучались в [15]. Эти решения соответствуют решениям с бесконечной энергией для уравнения (1.5) при отображении $\varphi \rightarrow i \varphi$, связываюшем указанные решения. Тем не менее формула связи для амплитуды $\gamma=\gamma(\alpha, \varphi)$ в (1.6) правильно воспроизводится этим отображением (см. [13]).

\section{2. РЕШЕНИЯ ТИПА СВЯЗАННОГО СОСТОЯНИЯ ДЛЯ УРАВНЕНИЯ РІІІ $(f=0)$}

2.1. Фазовые траектории. Уравнение (1.5) при $f=0$ представляет собой специальный случай третьего уравнения Пенлеве

$$
\frac{d^{2} \varphi}{d r^{2}}+\frac{1}{r} \frac{d \varphi}{d r}=\sin \varphi
$$

Его можно интерпретировать как уравнение нелинейного маятника с "временем" $r$ и зависящим от времени трением $r^{-1} \varphi_{r}$. Так же как и для классического маятника

$$
\frac{d^{2} \varphi}{d r^{2}}=\sin \varphi
$$

в фазовой плоскости $\left(\varphi, \varphi_{r}\right)$ выделяются седла в точках $(2 \pi k, 0), k=0, \pm 1, \pm 2, \ldots$, а центры в точках $(\pi+2 \pi k, 0)$ заменяются устойчивыми фокусами, обусловленными влиянием “трения" $r^{-1} \varphi_{r}$. Периодичность по сдвигу $\varphi \mapsto \varphi+2 \pi$ сохраняется, однако меняется поведение сепаратрис. Периодические сепаратрисы математического маятника $(2.2)$ удовлетворяют уравнению $\varphi_{r}^{2}=2(1-\cos \varphi)$, решения которого дают стандартные кинки-антикинки уравнения синус-Гордон

$$
\varphi(r)= \pm 4 \operatorname{arctg}\left[\exp \left(-\left(r-r_{0}\right)\right)\right]+2 \pi k .
$$




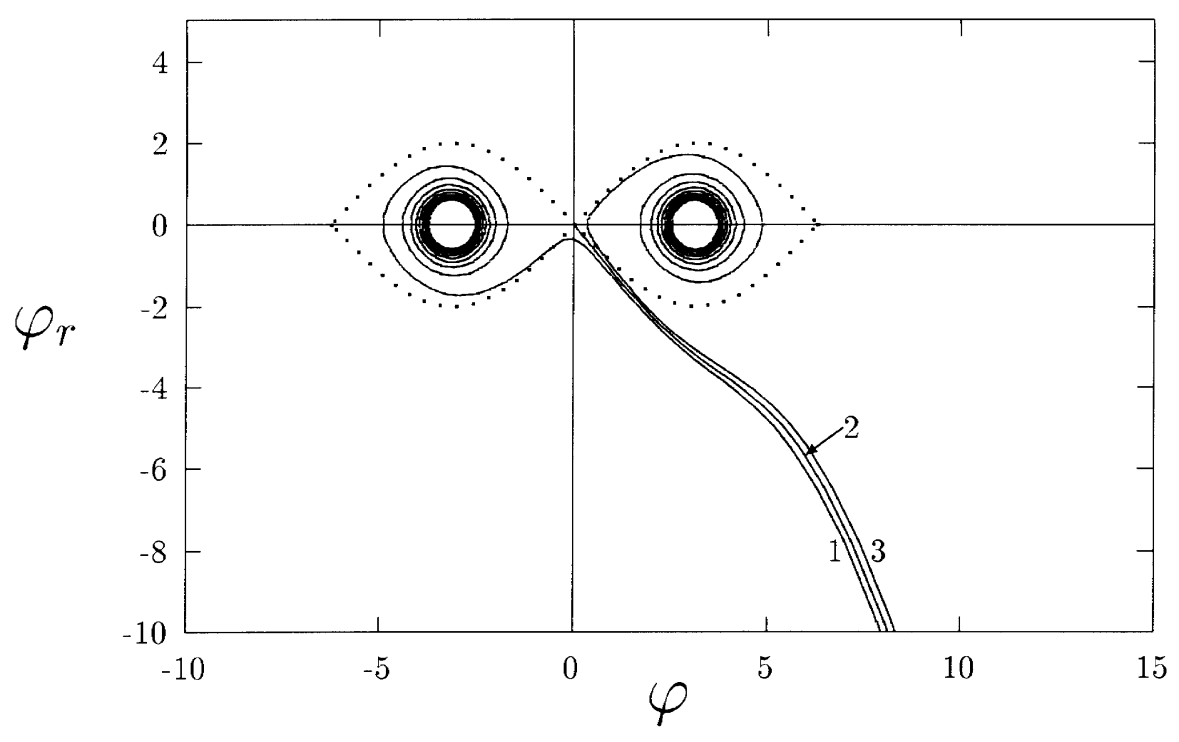

Рис. 1. Фазовые траектории для $\alpha=4$ и (1) $\beta=4.3$, (2) $\beta=4.497602$, (3) $\beta=4.7$. Пунктиром обозначены сепаратрисы классического маятника (2.2).

Вместо этих периодических кривых, нарисованных пунктиром на рис. 1, в уравнении (2.1) появляются уходящие на бесконечность “усы”, изображенные на рис. 1 сплошными линиями. Их асимптотики на бесконечности $(r \rightarrow 0)$ и в седловой точке $(r \rightarrow \infty)$ имеют вид (см. [13, 14])

$$
\begin{array}{ll}
\varphi(r)=\alpha \log \frac{1}{r}+\beta+O\left(r^{2}\right), & r \rightarrow 0, \\
\varphi(r)=2 \pi k+\gamma \sqrt{\frac{\pi}{2 r}} e^{-r}\left(1+O\left(r^{-1}\right)\right), & r \rightarrow \infty
\end{array}
$$

Остальные неособые фазовые траектории на бесконечности имеют асимптотику (2.4), в то время как при стремлении к фокусу -

$$
\varphi(r)=2 \pi(k+1 / 2)+\delta r^{-1 / 2} \cos \left\{r-\left(\delta^{2} / 16\right) \log (r)+\theta\right\}+o(1), \quad r \rightarrow \infty
$$

Ниже, в п. 2.2 будут приведены явные формулы, связывающие параметры асимптотик (2.4) и (2.5), (2.6). В промежуточной области (вне окрестности особых точек) фазовые траектории устроены достаточно нетривиально и не описываются простыми формулами. Поэтому интересующее нас поведение сепаратрисных решений исследовалось численно. Для интегрирования был использован метод пристрелки для стандартной схемы Рунге-Кутта четвертого порядка точности (см. [16]). Особенность при $r=0$ аппроксимировалась сдвигом начального условия (2.4) в близкую точку $r=r_{0} \ll 1$ :

$$
\varphi\left(r_{0}\right)=\alpha \log \frac{1}{r_{0}}+\beta, \quad \varphi_{r}\left(r_{0}\right)=-\frac{\alpha}{r_{0}} .
$$




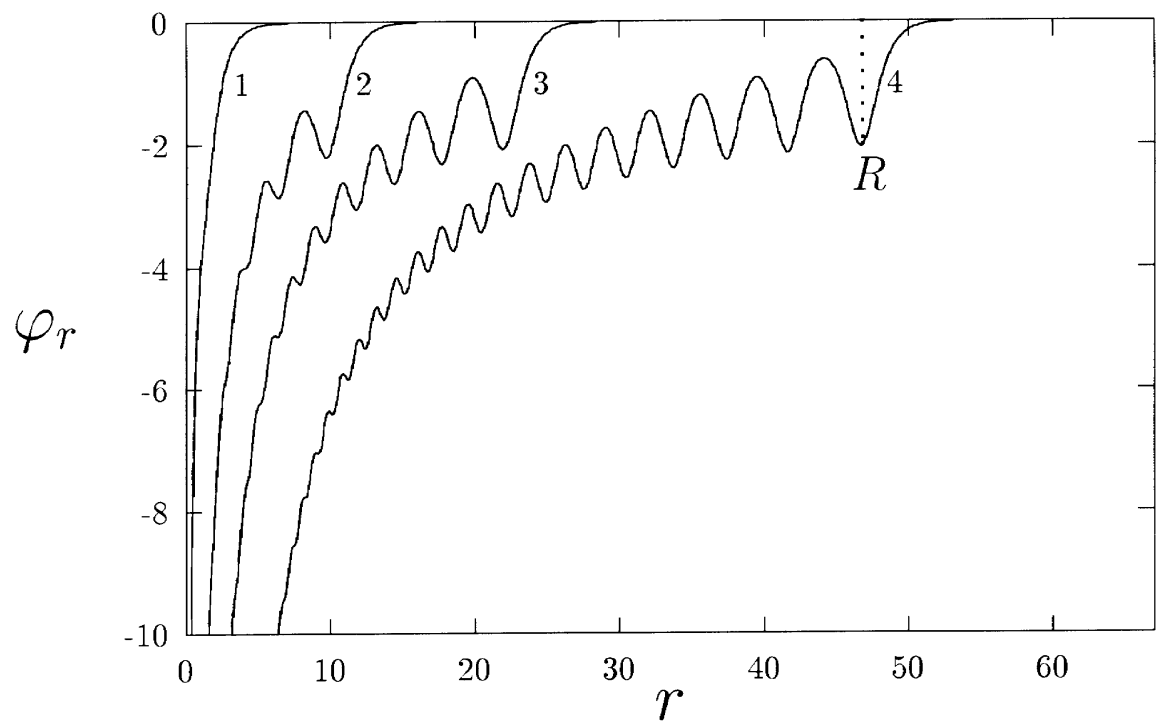

Рис. 2. Сепаратрисные решения $\varphi(r)$ уравнения PIII для (1) $\alpha=4,(2) \alpha=16$, (3) $\alpha=$ $32,(4) \alpha=64$.

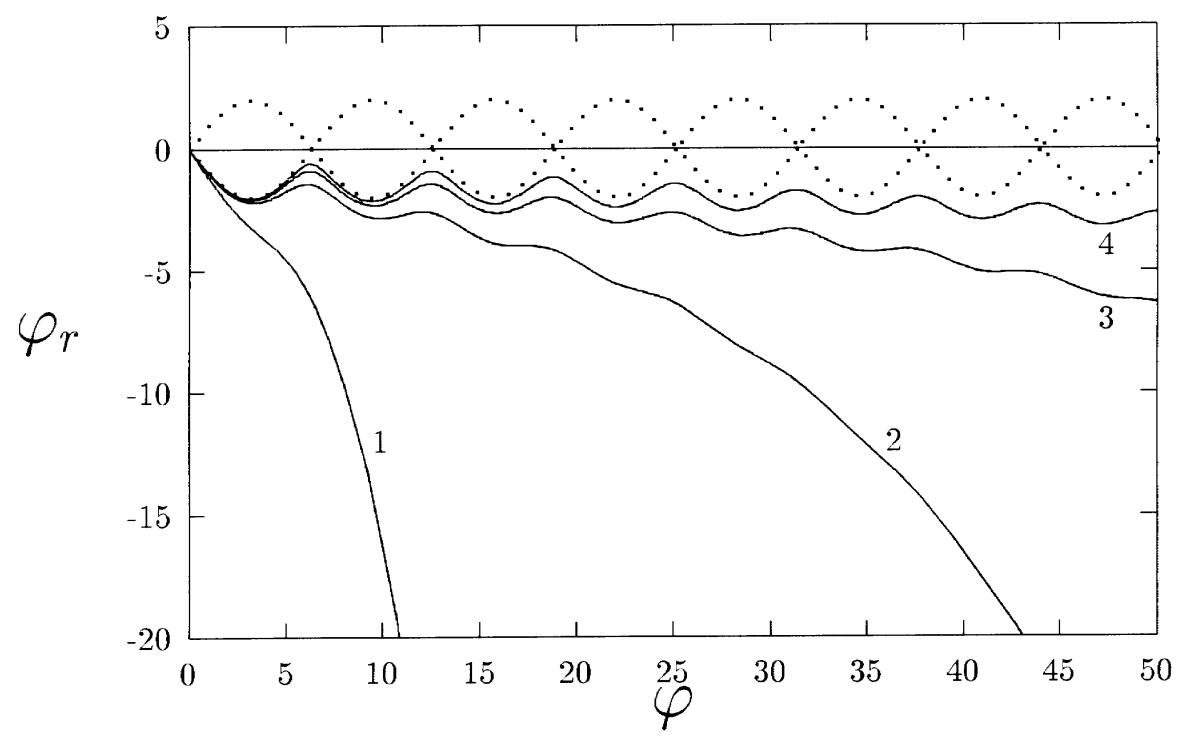

Рис. 3. Сепаратрисы уравнения РІІІ для (1) $\alpha=4$, (2) $\alpha=16$, (3) $\alpha=32$, (4) $\alpha=64$.

Корректность и сходимость этого приближения обоснованы в [17].

Указанный численный метод позволил подробно исследовать зависимость сепаратрисных решений от начального параметра $\alpha$. С ростом $\alpha$ осцилляции этих решений становятся все более выраженными (см. рис.2), что и дает радиальную структуру типа мишени для исходного двумерного уравнения (1.1). На рис. 3 видно, что соответствую- 


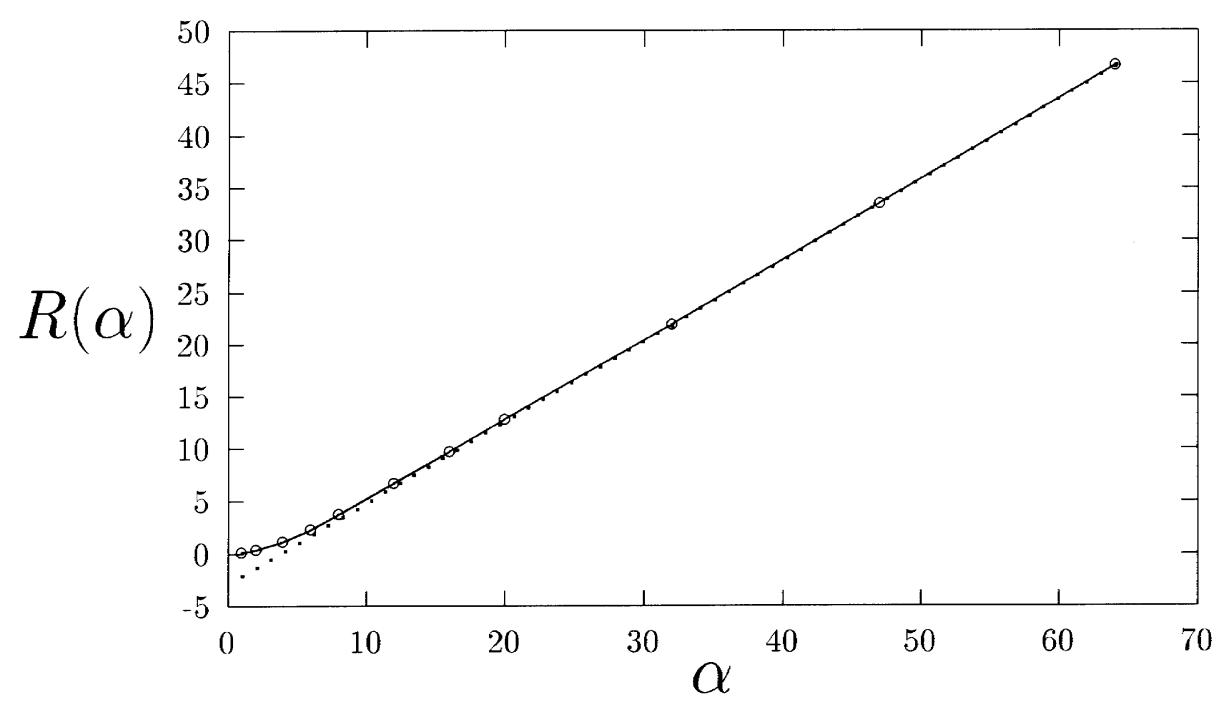

Рис. 4. Зависимость радиуса связанного состояния $R$ от $\alpha$ (сплошная линия) и приближение $R(\alpha) \approx(3.14 / 4) \alpha-2.90$ (пунктир).

щая сепаратриса становится более пологой с ростом $\alpha$ и стремится к сепаратрисе недиссипативного уравнения (2.2). Таким образом, для большого $\alpha$ и вдали от особенности связанные состояния действительно аппроксимируются квазипериодической решеткой хорошо различимых солитонов (2.3), которые подтверждают наблюдение, описанное в работах [2-5].

В приложениях для связанных состояний наиболее важен их эффективный радиус. Определим его следующим способом. Функция $\varphi(r)$ исчезает на бесконечности, так что координату $r=R$ назовем радиусом связанного состояния, если

$$
R=\sup \{r \mid \varphi(r)=\pi\} .
$$

Для большого $\alpha$ радиус $R$ может быть интерпретирован как координата самого удаленного изолированного минимума (см. рис. 2 для $\alpha=64)$, т.е. как координата последнего (внешнего) пика в солитонной решетке, за которым располагается область экспоненциального затухания.

Зависимость $R$ от $\alpha$ показана на рис. 4. Для большого $\alpha$ зависимость может быть аппроксимирована с достаточной точностью линейным соотношением

$$
R(\alpha) \approx C \alpha-2.90
$$

с численным значением коэффициента

$$
C \approx \frac{3.14}{4}
$$

2.2. Метод изомонодромных деформаций. Интегрирование уравнения PIII (2.1) методом изомонодромных деформаций предполагает рассмотрение следующей вспомогательной линейной системы:

$$
\frac{\partial \Psi}{\partial \lambda}=\left\{-\frac{r^{2}}{16} \sigma_{3}-\frac{i r \varphi_{r}}{4 \lambda} \sigma_{1}-\frac{1}{\lambda^{2}}\left(\sigma_{3} \cos \varphi-\sigma_{2} \sin \varphi\right)\right\} \Psi, \quad \lambda \in C,
$$


где $\Psi=\Psi(\lambda, r)-2 \times 2$-матрица, $\sigma_{1}, \sigma_{2}, \sigma_{3}$ - матрицы Паули, $r \in \mathbb{R}-$ фиксированный параметр, $\varphi=\varphi(r)$.

Система (2.10) содержит две иррегулярные особые точки $\lambda=0$ и $\lambda=\infty$. В окрестности этих точек определяется пара фундаментальных решений системы (2.10), нормированных асимптотиками

$$
\begin{array}{ll}
\Psi=\left(I+\Psi^{(1)}(r) \lambda^{-1}+O\left(\lambda^{-2}\right)\right) \exp \left(-\frac{i r^{2} \lambda}{16} \sigma_{3}\right), \quad \lambda \rightarrow \infty \\
\Phi=\left(\sin \frac{\varphi}{2}+i \sigma_{1} \cos \frac{\varphi}{2}\right)(I+O(\lambda)) \exp \left(\frac{i}{\lambda} \sigma_{3}\right), \quad \lambda \rightarrow 0 .
\end{array}
$$

Фундаментальные решения, в свою очередь, определяют матрицы монодромии системы (2.10) при обходе особых точек $\Psi\left(\lambda e^{2 \pi i}\right)=\Psi(\lambda) M_{1}, \Phi\left(\lambda e^{2 \pi i}\right)=\Phi(\lambda) M_{2}$, а также матрицу связи

$$
Q=\Phi^{-1}(\lambda) \Psi(\lambda)
$$

Ясно, что матришы $M_{1}, M_{2}$ и $Q$ не зависят от $\lambda$, поскольку $\Psi$ и $\Phi$ являются решениями одной и той же системы линейных уравнений. Вообще говоря, они зависят от параметров деформации $r$ и $\varphi$, однако их можно сделать постоянными по $r$ и $\varphi$, предполагая специальную зависимость $\varphi=\varphi(r)$. Это соотношение - условие изомонодромности деформации системы (2.10) - эквивалентно уравнению РІІІ на функцию $\varphi=\varphi(r)$ :

ТЕОрема 1 [12]. Функиия $\varphi=\varphi(r)$ удовлетворяет уравнению Пенлеве (2.1) тогда и только тогда, когда матрица связи $Q=\Phi^{-1} \Psi$ и матрицы монодромии для $\Phi$ u $\Psi$ при обходе особъх точек $\lambda=0$ и $\lambda=\infty$ не зависят от $x$.

В работах $[13,14]$ найдены простые алгебраические соотношения, выражающие матрицы монодромии $M_{1}, M_{2}$ через матрицу связи $Q$, так что матрица связи содержит полную информацию о решении уравнения Р III (2.1). Она может быть параметризована в виде

$$
Q=(-1)^{(k+1)}(1+p q)^{-1 / 2}\left(\begin{array}{cc}
1 & p \\
-q & 1
\end{array}\right),
$$

где параметры $p$ и $q$ являются в силу теоремы 1 первыми интегралами уравнения Пенлеве (2.1). Его решение $\varphi(r)$ восстанавливается (см. [12]) по фундаментальному решению линейной системы (2.10) по формуле

$$
\varphi_{r}(r)=-\frac{i r}{2} \Psi_{12}^{(1)}(r)
$$

где $\Psi_{12}^{(1)}$ - верхний внедиагональньй элемент коэффициента матрицы $\Psi^{(1)}$ в асимптотике (2.11).

Приведем кратко схему вычисления матрицы связи $Q$ по начальному условию $(2.4)$ в нуле. Подробности можно найти в монографии [14].

Подставим в систему (2.10) главный член асимптотики (2.5) $\varphi=-\alpha \log r+\beta$ и устремим $r$ к нулю. Поскольку $\alpha, \beta$ и $r$ вешественны, нетрудно показать, что матриша $\Psi$ при $|\lambda|>r^{-1 / 2}$ имеет вид

$$
\Psi=\psi+O\left\{\lambda^{-1} \exp \left(-i \sigma_{3} x^{2} \lambda / 16\right)\right\}
$$


где $\psi$ удовлетворяет укороченной системе (2.10)

$$
\frac{d \psi}{d \xi}=\left\{-i \sigma_{3}+\frac{i}{\alpha} 4 \xi \sigma_{1}\right\} \psi, \quad \xi=\frac{x^{2} \lambda}{16}
$$

Система (2.15) преобразуется к уравнениям Бесселя, откуда с учетом нормировки (2.11) находим

$$
\psi=\frac{1}{2} \sqrt{\frac{\pi}{2}} \exp \left(\frac{\pi \alpha}{8}\right) \xi^{1 / 2}\left(\begin{array}{cc}
-i H_{\nu}^{(2)}+H_{\nu-1}^{(2)} & i H_{-\nu+1}^{(1)}-H_{-\nu}^{(1)} \\
i H_{\nu}^{(2)}+H_{\nu-1}^{(2)} & i H_{-\nu+1}^{(1)}+H_{-\nu}^{(1)}
\end{array}\right)
$$

где $H_{\nu}=H_{\nu}(\xi)$ - функции Ханкеля первого и второго родов, $\nu=1 / 2+i \alpha / 4$.

Для второй матрицы (2.11) при $|\lambda|<r^{-3 / 2}$ справедливо представление

$$
\Phi=\phi+O\left\{x^{2} \lambda \exp \left(-i \sigma_{3} \lambda^{-1}\right)\right\}
$$

где $\phi$ удовлетворяет укороченной системе

$$
\frac{d \phi}{d \zeta}=\left\{i \sigma_{3} \cos \varphi-i \sigma_{2} \sin \varphi-\frac{i}{\alpha} 4 \zeta \sigma_{1}\right\} \phi, \quad \zeta=\lambda^{-1}
$$

Система (2.17) также преобразуется к уравнениям Бесселя, откуда, используя нормировку (2.11), находим

$$
\phi=\frac{1}{2} \sqrt{\frac{\pi}{2}} \exp \left(\frac{\pi \alpha}{8}\right) \zeta^{1 / 2}\left(\begin{array}{ll}
-i l H_{\nu}^{(2)}+l^{-1} H_{\nu-1}^{(2)} & i l^{-1} H_{-\nu+1}^{(1)}+l H_{-\nu}^{(1)} \\
-i l H_{\nu}^{(2)}-l^{-1} H_{\nu-1}^{(2)} & i l^{-1} H_{-\nu+1}^{(1)}+l H_{-\nu}^{(1)}
\end{array}\right)
$$

где $H_{\nu}=H_{\nu}(\zeta), l=\exp (-i \beta / 2-i \pi / 2), \nu=1 / 2+i \alpha / 4$.

В области $r^{-1 / 2}<|\lambda|<r^{-3 / 2}$ остаточные члены $\Psi-\psi$ и $\Phi-\phi$ малы одновременно, так что представления (2.16) и (2.18) можно использовать для вычисления главного члена асимптотики $Q$ при $r \rightarrow 0$. Поскольку в этой области переменные $\xi, \zeta$ малы, следует подставить в $(2.16)$ и $(2.18)$ вместо $H_{\nu}$ их асимптотики при $\xi, \zeta \rightarrow 0$. Поскольку матрица $Q$ не зависит от $x$, ее асимптотика при $r \rightarrow 0$, вычисленная таким образом, совпадает с точной матрищей $Q$ :

$$
Q=\frac{1}{2 \pi i}\left(\begin{array}{cc}
A+B & A e^{\pi \alpha / 4}-B e^{-\pi \alpha / 4} \\
A e^{-\pi \alpha / 4}-B e^{\pi \alpha / 4} & A+B
\end{array}\right)
$$

где

$$
A=i 2^{-3 i \alpha / 2} e^{i \beta / 2} \Gamma^{2}\left(\frac{1}{2}-\frac{i \alpha}{4}\right), \quad B=-i 2^{3 i \alpha / 2} e^{-i \beta / 2} \Gamma^{2}\left(\frac{1}{2}+\frac{i \alpha}{2}\right)
$$


2.3. Формулы связи. Приступим теперь к вычислению асимптотики сепаратрисного решения с асимптотикой (2.5) на бесконечности. Оказывается [13], что это решение выделяется условиями

$$
\begin{gathered}
q=0, \quad p=-2 \sinh \frac{\pi \alpha}{4} \\
e^{i \beta}=2^{3 i \alpha} \Gamma^{-2}\left(\frac{1}{2}-\frac{i \alpha}{4}\right) \Gamma^{2}\left(\frac{1}{2}+\frac{i \alpha}{4}\right) e^{\pi \alpha / 2}
\end{gathered}
$$

Структура матрицы $Q$ в этом случае $\left(Q=\sigma_{1}\right)$ позволяет переформулировать задачу монодромии (2.12) как задачу Римана о факторизации матриц на вешественной оси в комплексной $\lambda$-плоскости:

$$
\Psi_{+}(\lambda, r)=\Psi_{-}(\lambda, r) G(\lambda), \quad \lambda \in \mathbb{R},
$$

где $\Psi_{+}$и $\Psi_{-}-$аналитические функции в верхней $(\operatorname{Im} \lambda>0)$ и нижней $(\operatorname{Im} \lambda<0)$ полуплоскостях с асимптотиками $(2.11)$, соответственно. Матрица $G(\lambda)$ имеет вид ${ }^{1)}$

$$
G(\lambda)= \begin{cases}\left(\begin{array}{cc}
1 & -p \\
0 & 1
\end{array}\right), & \lambda>0 \\
\left(\begin{array}{cc}
1 & 0 \\
p & 1
\end{array}\right), & \lambda<0 .\end{cases}
$$

Задача (2.23) сводится к системе сингулярных интегральных уравнений

$$
\chi_{+}(\lambda, r)=I+\frac{1}{2 \pi i} \int_{-\infty}^{\infty} \frac{\chi_{+}(\eta, r)}{\eta-\lambda-i 0} e^{\frac{i r^{2}}{16} \lambda \sigma_{3}-\frac{i}{\lambda} \sigma_{3}}(I-G(\lambda)) e^{-\frac{i r^{2}}{16} \lambda \sigma_{3}+\frac{i}{\lambda} \sigma_{3}} d \eta
$$

где

$$
\chi_{ \pm}(\lambda, r)=\Psi_{ \pm}(\lambda, r) e^{\frac{i r^{2}}{16} \lambda \sigma_{3}-\frac{i}{\lambda} \sigma_{3}}
$$

Для первой строки матрицы $\chi$ получаются скалярные уравнения

$$
\begin{aligned}
& \chi_{11}=1+\frac{1}{2 \pi i} \int_{-\infty}^{0} \frac{p \chi_{12}(\eta, r)}{\eta-\lambda-i 0} e^{\frac{i r^{2}}{8} \eta-\frac{2 i}{\eta}} d \eta, \\
& \chi_{12}=\frac{1}{2 \pi i} \int_{0}^{\infty} \frac{-p \chi_{11}(\eta, r)}{\eta-\lambda-i 0} e^{-\frac{i r^{2}}{8} \eta+\frac{2 i}{\eta}} d \eta .
\end{aligned}
$$

Асимптотика решений системы (2.25), (2.26) при $r \rightarrow \infty$ получается применением метода перевала. Поскольку $\chi_{12}$ аналитична в верхней полуплоскости, контур интегрирования может быть деформирован вверх так, чтобы он прошел через седловую точку

$$
\eta_{\mathrm{st}}=\frac{4 i}{r}
$$

\footnotetext{
${ }^{1)}$ Подробности вычислений см. в $[13,14]$.
} 
Отсюда имеем асимптотическую оценку $\chi_{11}=1+O\left(r^{-1 / 2} e^{-r}\right)$. Подставляя ее в уравнение (2.26), получаем

$$
\chi_{12}(\lambda, r)=-\frac{p}{2 \pi i} \int_{0}^{\infty} \frac{e^{-\frac{8}{\eta} i r^{2}+\frac{2 i}{\eta}}}{\eta-\lambda-i 0} d \eta+O\left(r^{-1} e^{-r}\right), \quad r \rightarrow \infty .
$$

Снова применим к этому уравнению метод перевала, сдвигая контур в нижнюю полуплоскость $(\operatorname{Im} \lambda<0)$ и делая замены $\lambda=\zeta / r, \eta=\xi / r$. Окончательно приходим к асимптотике

$$
\chi_{12}(\lambda, r)=-\frac{4 i p}{\sqrt{2 \pi}} r^{-3 / 2} e^{-r} \lambda^{-1}+O\left(\lambda^{-2}\right), \quad \lambda \rightarrow \infty .
$$

В результате по формуле обрашения (2.14) получаем

$$
\varphi_{r}(r)=-p \sqrt{\frac{2}{\pi r}} e^{-r}+O\left(r^{-1} e^{-r}\right), \quad r \rightarrow \infty .
$$

Интегрируя по $r$ и используя (2.21), получаем асимптотику (1.5), где константа $\gamma$ равна

$$
\gamma=\frac{4}{\pi} \sinh \frac{\pi \alpha}{4} .
$$

Целую константу $k$ - номер седловой точки, в которую входит сепаратриса, - также можно вычислить с помощью метода изомонодромных деформаций. Для этого достаточно найти формулы связи для обшего (несепаратрисного) решения с асимптотикой (2.6). Не вдаваясь в детали вычислений, приведем лишь окончательный результат.

Теорема 2 [13]. Общее двухпараметрическое вещественное рещение уравнения PIII (2.1) с начальным условием (2.4) имеет асимптотику (2.6) при $r \rightarrow \infty$, где

$$
\begin{gathered}
\delta^{2}=-\frac{16}{\pi} \log \left(\frac{1}{\pi}|\operatorname{Re} A|\right), \quad|\operatorname{Re} A| \neq 0, \\
\theta=\frac{\pi}{4}+\arg \Gamma\left(\frac{i \delta^{2}}{16}\right)-\frac{\delta^{2}}{8}+\arg \left(A e^{-\pi \alpha / 4}-B e^{\pi \alpha / 4}\right),
\end{gathered}
$$

А и В заданьц формулами (2.20).

Определим теперь значение целочисленного параметра $k=k(\alpha, \beta)$ в асимптотике (2.6). Очевидно, что равенства $k(\alpha, \beta)=n, n \in \mathbb{Z}$, разбивают плоскость начальных данных $\alpha, \beta$ на счетное число областей. Когда точка $(\alpha, \beta)$ стремится к границе любой из областей, параметры асимптотики (2.6) терпят разрыв. Формулы (2.28) показывают, что разрыв имеет место только при $\operatorname{Re} A=0$, откуда

$$
\beta-3 \alpha \log 2+4 \arg \Gamma\left(\frac{1}{2}-\frac{i \alpha}{4}\right)=2 \pi k .
$$

Равенство (2.29) определяет границы областей постоянного $k$. При $\alpha=0$ ограниченное решение уравнения PIII (2.1) с начальным условием $\varphi(0)=\beta$ заключено в интервале $2 \pi k<\varphi<2 \pi(k+1)$, где $k=\left[\frac{\beta}{2 p i}\right],[\cdot]$ - целая часть числа. Поскольку каждая область с гранишами (2.29) пересекается прямой $\alpha=0$ один раз, получаем из (2.29) выражение для $k$ при всех $\alpha$ : 
СЛЕДСТВИЕ ТЕОРЕМЫ 2. Постоянная $k$ в асимптотике (2.1) равна

$$
k=\left[\frac{1}{2 \pi}\left(\beta-3 \alpha \log 2+4 \arg \Gamma\left(\frac{1}{2}-\frac{i \alpha}{4}\right)\right)\right] .
$$

Формулы связи (2.21), (2.27) и (2.30) полностью определяют асимптотики (1.2), (1.6) сепаратрисного решения уравнения PIII (2.1). К сожалению, промежуточный осциллирующий режим этого решения, показанный на рис. 2 , не допускает столь же эффективного аналитического описания. Тем не менее полученные формулы связи позволяют обосновать наблюдаемую численно зависимость (2.8) радиуса связанного состояния от параметра $\alpha$.

Фиксируем $\varphi=$ const в асимптотической формуле (1.6), тогда уравнение

$$
\frac{4}{\pi} \sinh \left(\frac{\pi \alpha}{4}\right) \sqrt{\frac{\pi}{2 R}} e^{-R}=\mathrm{const}
$$

определяет границу $R(\alpha)$ области колебаний, где выполнено условие $\varphi(R)=$ const. Из уравнения (2.31) получаем при $R \gg 1$

$$
R(\alpha)=\frac{\pi}{4} \alpha-\frac{1}{2} \log \alpha+O(1), \quad \alpha \rightarrow \infty .
$$

Это соотношение хорошо согласуется с (2.8) и (2.9), поскольку $d R / d \alpha$ при больших $\alpha$ слабо зависит от $\alpha$.

\section{3. ЭЛЛИПТИЧЕСКОЕ УРАВНЕНИЕ СИНУС-ГОР ДОН С ПРАВОЙ ЧАСТЬЮ}

Найдем условия существования и устойчивости решений типа связанных состояний для неоднородного уравнения синус-Гордон (1.1) с правой частью $f$. Будем рассматривать его как возмушение интегрируемого случая $f=0$, что позволяет при малых возмущениях применять аналитическую теорию, развитую в п. 2.2. Однако в интересующем нас случае больших возмушений неинтегрируемость уравнения (1.1) заставляет прибегнуть к численным методам, описанным в п. 2.1.

Ясно, что возмушение $f$ не оказывает влияния на особенность (1.2) в нуле:

$$
\varphi(r)=\alpha \log \frac{1}{r}+\beta+O\left(r^{2}\right), \quad r \rightarrow 0
$$

На бесконечности $r \rightarrow \infty$ асимптотика связанного состояния принимает вид

$$
\varphi(r)=\varphi_{0}+\gamma \sqrt{\frac{\pi}{2 r}} e^{-r}\left(1+O\left(r^{-1}\right)\right), \quad \sin \left(\varphi_{0}\right)=f
$$

Тем самым седловые точки сдвигаются на $\left(\varphi_{0}, 0\right)$. Это дает необходимое условие на $f$ для существования связанных состояний в уравнении (1.5): $|f| \leq 1$.

Ограничимся случаем $\alpha>0$, так как отрицательные $\alpha$ получаются простым изменением знаков в $(1.5): \varphi \rightarrow-\varphi$ и $f \rightarrow-f$. Ясно, что малое возмушение $f \ll 1$ не разрушает связанное состояние, удовлетворяюшее (3.2). Однако сушествует критическое значение 


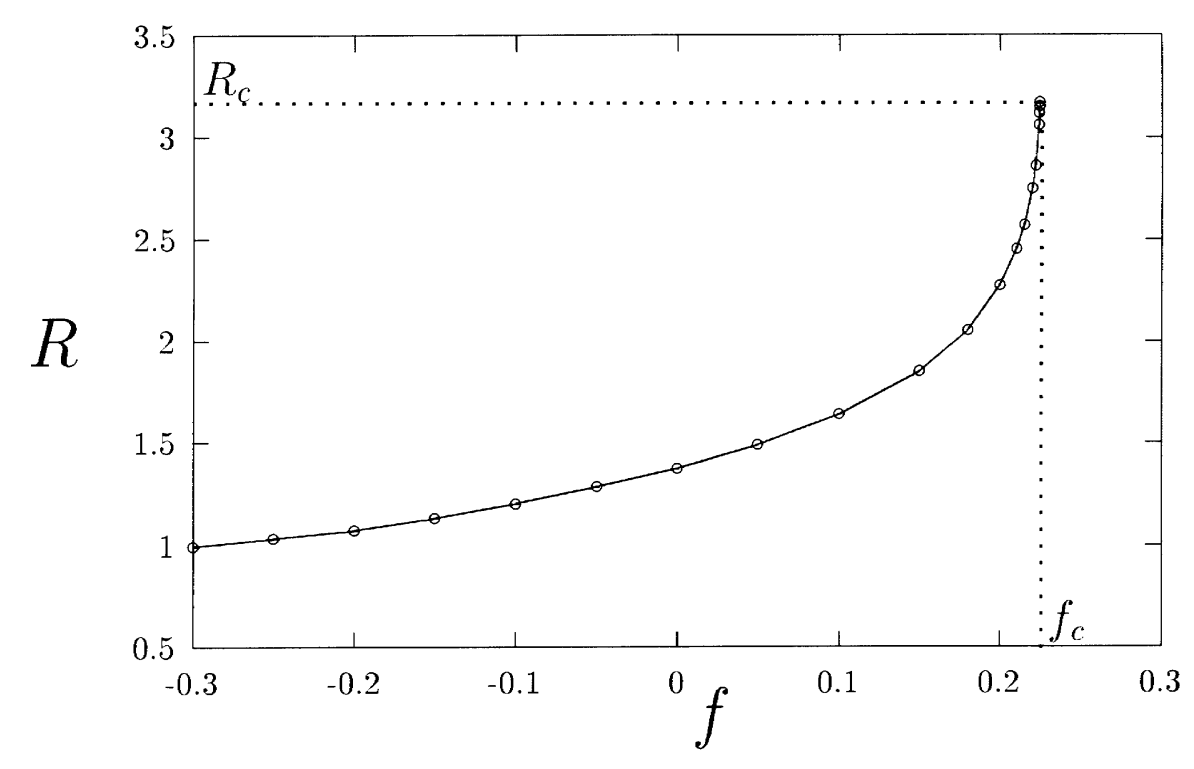

Рис. 5. Зависимость радиуса связанного состояния $R$ от возбуждения $f$ для $\alpha=4$.

$0<f_{c}(\alpha)<1$, вне которого не найдется решений с асимптотиками (3.1) и (3.2). Для больших $\alpha$ его численная оценка дается формулой

$$
f_{c}(\alpha) \approx \frac{A}{\alpha}
$$

с константой $A \approx 0.80887$.

При $f \leq f_{c}(\alpha)$ качественная структура решений подобна обсуждавшейся вьше в п. 2.1, причем радиус связанного состояния $R$ становится зависящим от $f$ (см. рис.5). Эта зависимость монотонна вплоть до критического значения $f_{c}$, отвечающего некоторому значению $R_{c}$. Зависимость критического радиуса $R_{c}$ от $\alpha$ почти линейна при больших $\alpha \gg 1$. Она приближенно имеет вид

$$
R_{c} \approx B \alpha,
$$

где константа $B \approx 1.52$.

На рис. 6 показаны решения вблизи от критического состояния $\left(f \approx f_{c}\right.$ и $\left.R \approx R_{c}\right)$ для различных $\alpha$. Сравнение с решениями невозмушенного уравнения PIII (2.1), изображенными на рис. 2 , показывает значительное расширение солитонной решетки, а также распадение ее на отдельные солитоны во внешней области. Пространственное разделение внешних солитонов с ростом $\alpha$ позволяет предложить простую физическую модель, ответственную за разрушение связанного состояния при $f>f_{c}$.

Модель основана на адиабатической теории возмушения для радиально-симметричных солитонов уравнения синус-Гордон $(1.5)[18,19]$, где члены $r^{-1} \varphi_{r}$ и $f$ могут рассматриваться как малые возмушения порядка $O(1 / \alpha)$. Рассмотрим одиночный кольцевой солитон типа кинка (2.3) с большим радиусом $r_{0} \gg 1$. Подстановка (2.3) в (1.3) дает энергию солитона

$$
E_{s} \approx 16 \pi r_{0}-2 \pi^{2} f r_{0}^{2}+O(1 / \alpha),
$$




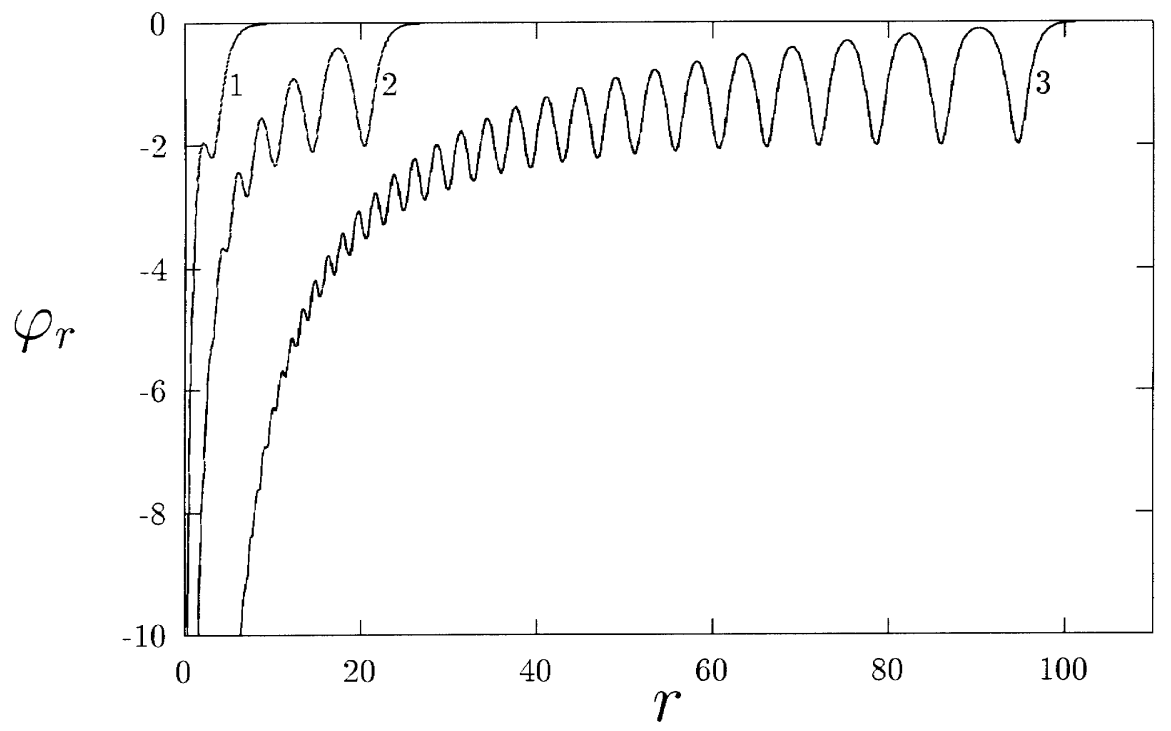

Рис. 6. Связанные состояния $\varphi(r)$ для уравнения (1.5) с правой частью $f$ вблизи критического значения $f_{c}$ : (1) $\alpha=4, f_{c}=0.22433 ;(2) \alpha=16, f_{c}=0.051225$; (3) $\alpha=64$, $f_{c}=0.012680$.

которая имеет экстремум в точке

$$
R_{0 e} \approx \frac{4}{\pi f} .
$$

При условии малости диссипации $r^{-1} \varphi_{r}$ аппроксимация связанного состояния кинком (2.3) справедлива в окрестности $r_{0}=r_{0 e} \gg 1$. Но она неустойчива, поскольку соответствует максимуму энергии в (3.5). Принимая (2.3) в качестве начального условия для эволюционного уравнения (1.4), получаем, что возникающее в результате решение $\varphi(r, t)$ коллапсирует к центру $r=0$ при $r_{0}<r_{0 e}$ или стремится к бесконечности при $r_{0}>r_{0 e}$. Возвращаясь к связанным состояниям уравнения $(1.5)$, заключаем, что при $R<r_{0 e}$ коллапс солитонной решетки предотврашается влиянием особенности (1.2), которая действует как отталкивающий центр. Тем самым решение типа связанного состояния сушествует, пока его радиус, возрастающий вместе с $f$, не достигнет значения $R \approx r_{0 e}$. Далее, можно ожидать, что связанное состояние будет разрушено неустойчивостью внешнего кольцевого солитона. Формулы (3.3) и (3.4), полученные численным моделированием, дают зависимость

$$
R_{c} \approx \frac{H}{f_{c}},
$$

где $H \approx 1.23$. Это находится в хорошем согласии с (3.6), подтверждая нашу адиабатическую теорию критического поведения.

\section{4. ДВА ПРИЛОЖКЕНИЯ}

4.1. Контакт Джозефсона большой площади. Уравнение синус-Гордон (1.1) является обшепринятой моделью двумерного контакта Джозефсона с размерами, намного большими джозефсоновской глубины проникновения $\lambda_{J}$ (см. [20]). Здесь $\varphi(x, y)-$ 
распределение разности фаз между сверхпроводящими электродами, а $f$ - ток смещения, однородно распределенный по области перехода. Безразмерное уравнение (1.1) получается после перенормировки координат на величину $\lambda_{J}$. Кроме того, будем допускать сингулярные решения, отвечаюшие дельтообразным возмущениям правой части. Тогда решения типа связанного состояния с особенностью в начале координат описываются уравнением

$$
\Delta \varphi=\sin \varphi-f-2 \pi \alpha \delta(\mathbf{r})
$$

и приобретают ясный физический смысл как отклик на включение точечного тока $j=-2 \pi \alpha$ [21]. Появление таких дельтообразных токов смещения невозможно исключить в широких токонесущих электродах, где велико влияние неоднородностей $[1,2]$, в то время как от гладкой компоненты тока можно избавиться благодаря некоторым структурным особенностям двумерных контактов [20].

В физике джозефсоновских переходов солитоны (2.3) принято называть флюксонами или вихревыми линиями квантованного потока. Поскольку фундаментальное решение уравнения (4.1) имеет логарифмическую асимптотику (1.2), для нулевых граничных условий решение $\varphi(x, y)$ конечной энергии должно иметь вид связанного состояния $(2.4),(2.5)$. Естественно ожидать поэтому появления структуры типа кольцевых флюксонов с центром в точке сингулярности тока смешения и радиусом $R$, заданным формулой (2.8). Будучи решениями уравнения PIII (2.1), они устойчивы для произвольного $\alpha$, так как обычно $f=0$ в двумерных контактах. Неустойчивость возникает только из-за влияния границ контакта или при взаимодействии флюксонов $[2,3]$. Обсудим ее на простой модели прямоугольного контакта, которая предложена в $[1,2]$.

Пусть для простоты область перехода есть квадрат со стороной $L \gg 1$. Граничньй ток наблюдается прежде всего вблизи углов, и, как показано в [1], плотность его хорошо аппроксимируется дельтообразными сингулярностями в вершинах квадрата. Таким образом, в уравнении (4.1) следует поместить источники в вершины квадрата и дополнить его условием Неймана на границе

$$
\partial \varphi / \partial \mathbf{n}=0
$$

Условие (4.2) отвечает отсутствию тока вне углов. Рассмотрим простейший случай равенства токов $j_{m}=-2 \pi \alpha_{m}, \alpha_{m}=\alpha, m=1,2,3,4$, во всех вершинах, который исследовался численно в [3].

Для малых $\alpha$ радиус связанного состояния мал: $R(\alpha) \ll L$, и распределение фазы вблизи угла хорошо аппроксимируется решениями РІІІ благодаря граничным условиям Неймана (4.2). Радиус $R$ увеличивается с ростом $\alpha$, и для некоторого критического значения $\alpha=\alpha_{c}$, когда $R \approx L / 2$, связанные состояния от различных углов начинают взаимодействовать, что приводит к неустойчивости. Соответствующий критический ток $j_{c}=-2 \pi \alpha_{c}$ есть наиболее важная характеристика джозефсоновского перехода, поскольку он определяет разрушение стационарного сверхпроводяшего состояния и приводит к динамическому состоянию, имеющему ненулевое сопротивление [20]. Из уравнений $(2.8)$ и $(2.29)$, полагая $R\left(\alpha_{c}\right) \approx L / 2$, можно получить критическое значение $\alpha$

$$
\alpha_{c} \approx \frac{2}{\pi} L
$$

которое находится в хорошем согласии с компютерным вычислением критического значения в [3]. 
4.2. Жидкие кристаллы. Формирование структур типа мишени было обнаружено в недавних экспериментах $[4,5]$ с нематиками, размешенными во врашающемся магнитном поле. В экспериментах тонкая пленка нематика была зажата между двумя стеклянными дисками, которые помещались во вращающееся магнитное поле с частотой $\omega$ и вектором $\mathbf{H}$, лежашим в плоскости пленки ( $x y$-плоскость):

$$
\mathbf{H}=\left(H_{0} \cos \omega t, H_{0} \sin \omega t, 0\right) \text {. }
$$

Значение $H_{0}$ намного превьшало поле перехода Фредерикса, следовательно, директор $\mathbf{n}$ лежал в основном в $(x y)$-плоскости. Уравнение движения для $\mathbf{n}(x, y, t) \quad\left(\mathbf{n}^{2}=1\right)$ имеет вид $[22,6]$

$$
\gamma_{1} \mathbf{n}_{t}=K[\Delta \mathbf{n}-\mathbf{n}(\mathbf{n} \cdot \Delta \mathbf{n})]+\chi_{a}(\mathbf{n} \cdot \mathbf{H})(\mathbf{H}-\mathbf{n}(\mathbf{n} \cdot \mathbf{H})),
$$

где $\gamma_{1}$ - врашательная вязкость, $\chi_{a}>0$ - анизотропия диамагнитной восприимчивости, а $K=K_{1}=K_{3}$ (приближение с одной константой анизотропии). Полагая $\mathbf{n}=(\cos \varphi(x, y, t), \sin \varphi(x, y, t), 0)$ и используя замену $(4.4)$, перепишем уравнение $(4.5)$ в виде

$$
\gamma_{1} \varphi_{t}=K \Delta \varphi-\chi_{a} H_{0}^{2} \sin (\varphi-\omega t) \cos (\varphi-\omega t) .
$$

Переходя, далее, к врашаюшейся системе координат $\varphi \mapsto \varphi-\omega t$ и к безразмерным переменным, приводим уравнение (4.6) к нестационарному уравнению синус-Гордон (1.4)

$$
-\varphi_{t}+\Delta \varphi=\sin 2 \varphi-f
$$

для угла $\varphi(x, y, t)$ между $\mathbf{n}$ и $\mathbf{H}(t)^{2)}$, где $f=2 \omega \gamma_{1} / \chi_{a} H_{0}^{2}$.

Для стационарных решений выполнено уравнение

$$
\Delta \varphi=\sin 2 \varphi-f
$$

Связанные состояния, как показано в разделе 3 , существуют только для $|f| \leq 1$ и уравнение

$$
\sin 2 \varphi_{0}-f=0
$$

определяет однородное состояние, когда директор $\mathbf{n}_{0}$ врашается синхронно с полем $\mathbf{H}$ и отстает от него на угол $\varphi_{0}$.

Физическая интерпретация связанных состояний в этой модели заключается в следуюшем. Рассмотрим вариацию свободной энергии для уравнения (4.8)

$$
F=\int\left\{\frac{1}{2}(\nabla \varphi)^{2}-\frac{1}{2} \cos 2 \varphi+\frac{1}{2} \cos 2 \varphi_{0}-f\left(\varphi-\varphi_{0}\right)\right\} d V
$$

в кольцевой области $D$ с центром в сингулярной точке, полагая внутренний радиус $a \ll 1$ и обозначая произвольньй внешний радиус через $a^{\prime}$ :

$$
\delta F=\int_{D}\{(\nabla \varphi \cdot \nabla \delta \varphi)+\sin 2 \varphi \cdot \delta \varphi-f \cdot \delta \varphi\} d V
$$

\footnotetext{
${ }^{2)}$ Множитель 2 в $\sin 2 \varphi$ может быть легко исключен заменами $t \rightarrow 2 t, \mathbf{r} \rightarrow \sqrt{\mathbf{2}} \mathbf{r}$, но мы сохраняем его для физического значения угла $\varphi$.
} 
Перейдем здесь к интегрированию по границе и устремим внешний радиус к бесконечности: $a^{\prime} \rightarrow \infty$. Интеграл по внешней окружности исчезнет из-за быстрой сходимости $\varphi$ к однородному состоянию $(4.9)$ и $\delta \varphi \rightarrow 0$. Окончательно имеем

$$
\delta F=\int_{a} \delta \varphi(\nabla \varphi \cdot d \mathbf{s})
$$

а поскольку на внутренней окружности $\nabla \varphi \approx \nabla(\alpha \log (1 / r))$, получим

$$
\delta F \approx 2 \pi \alpha \delta \varphi_{a}
$$

Здесь $\delta \varphi_{a}$ - угол вращения директора $\mathbf{n}$ в круге $a$. Вариация свободной энергии относительно врашения соответствует работе некоторого момента сил $\boldsymbol{\Gamma}$, действующего на нематик. Его z-компонента равна

$$
\Gamma_{z} \approx 2 \pi \alpha
$$

где $\alpha$ есть коэффициент при логарифме в сингулярной асимптотике (1.2).

Появление подобных локализованных возмущений обычно связывается с маленькими частицами пыли, плаваюшими в нематике [22, 23]. Асимптотика (1.2) здесь описывает дальнодействующую деформацию профиля $\varphi$, вызванную частицей $[4,5]$. Детальная оценка вида $\Gamma_{z}$ вблизи частицы выходит за рамки нашего исследования. Отметим лишь, что в случае асимметричной частицы, вытянутой в направлении $\mathbf{N}$ (направление главной оси эллипсоидной частишы), обший вид $\Gamma_{z}$ был найден в [23]:

$$
\Gamma_{z} \approx b(\cos \theta) \sin \theta
$$

с некоторой нечетной функцией $b(\xi)$ и углом $\theta$ между $\mathbf{N}$ и $\mathbf{n}_{0}$. Врашательная сила, таким образом, исчезает, когда $\mathbf{N}$ и $\mathbf{n}_{0}$ параллельны $(\theta=0, \pi)$.

В случае нематиков со свободно плавающими частицами без магнитного поля минимум свободной энергии достигается для $\alpha \sim \Gamma_{z}=0$, так что никакой радиально-симметричной структуры не возникает. В этом случае дефекты типа диполя - единственно возможные состояния [21]. С другой стороны, при включении поля $\mathbf{H} \neq 0$ вращающийся директор $\mathbf{n}$ возбуждает врашение свободно плаваюшей частицы, и можно надеяться, что для достаточно малых $\omega$ возможно синхронное врашение частицы, но с другим углом $\varphi_{0}^{\prime}$ из-за различной эффективной вращательной вязкости для макроскопической частицы. Таким образом, во вращаюшемся магнитном поле устанавливается постоянньй сдвиг $\theta=\varphi_{0}-\varphi_{0}^{\prime}$, который дает ненулевой момент сил, и $\alpha \neq 0$.

Как показано в разделе 3 , связанные состояния сушествуют и устойчивы только для $\alpha f \leq 0$ или для $\alpha f>0$ и $|f|<f_{c}$. Если $f$ превышает критическое значение $f_{c}$, они становятся нестабильны и согласно адиабатической теории раздела 3 можно ожидать возникновения нового динамического состояния. Это явление было исследовано компютерным моделированием уравнения (4.7) [3, 7] и качественно согласуется с экспериментами $[4,5]$. Было установлено, что свободная частица действует как центр эмиссии ("ведущий" центр) кольцевых солитонов. Вдали от центра они формируют структуру типа мишени с границей, удаляюшейся от центра с некоторой предельной скоростью, которая определена конкуренцией между возбуждением и диссипацией.

Работа выполнена при поддержке РФФИ, грант № 96-01-00128. 


\section{Список литературы}

[1] A. Barone, F. Esposito, K. K. Likharev, V. K. Semenov, B. N. Todorov, R. Vaglio. J. Appl. Phys. 1982. V. 53. P. 5802.

[2] П. Н. Вабищевич, С.А. Васенко, К.К. Лихарев, В.К. Семенов. ЖЭТФ. 1984. Т. 86. C. 1132 .

[3] A. G. Shagalov. In: Nonlinear Superconducting Devices and High- $T_{c}$ Materials. Ed. R. D. Parmentier and N. F. Pedersen. Singapore: World Scientific, 1995. P.125.

[4] K. B. Migler, R. B. Meyer. Phys. Rev. Lett. 1991. V. 66. P. 1485.

[5] K. B. Migler, R. B. Meyer. Physica D. 1994. V. 71. P. 412.

[6] T. Frisch. Physica D. 1995. V. 84. P. 601.

[7] A. G. Shagalov. Phys. Lett. A. 1995. V. 199. P. 229.

[8] T. Frisch, S. Rica, P. Coullet, J. M. Gilli. Phys. Rev. Lett. 1994. V. 72. P. 1471.

[9] G. H. Derrick. J. Math. Phys. 1964. V. 5. P. 1252.

[10] V. G. Makhankov. Phys. Rep. 1978. V. 35. P. 1.

[11] Л. Д. Ландау, Е. М. Лифииц. Теория упругости. М.: Наука, 1987.

[12] H. Flaschka, A. Newell. Commun. Math. Phys. 1980. V. 76. P. 67.

[13] В. Ю. Новокшенов. ДАН СССР. 1985. Т. 283. № 5. С. 1161.

[14] A.R. Its, V. Yu. Novokshenov. The isomonodromic deformation method in the theory of Painlevé equations. In: Lecture Notes in Mathematics. V. 1191. Berlin, Heidelberg, New York: Springer-Verlag, 1986.

[15] B. M. McCoy, C. A. Tracy, T. T. Wu. J. Math. Phys. 1977. V. 18. P. 1058.

[16] W. H. Press, S. A. Teukolsky, W. T. Vetterling, B.P. Flannerty. Numerical Recipes in FORTRAN. Cambridge: Cambridge University Press, 1992.

[17] А.А. Абрамов. ЖВМи МФ. 1980. Т. 20. С. 1155.

[18] E. M. Maslov. Physica D. 1985. V. 15. P. 433.

[19] E. M. Maslov. Phys. Lett. A. 1988. V. 131. P. 364.

[20] A. Barone, G. Paterno. Physics and Applications of the Josephson Effect. New York: Academic Press, 1982.

[21] A. G. Shagalov. Phys. Lett. A. 1992. V. 165. P. 412.

[22] P. G. de Gennes. The Physics of Liquid Crystals. Oxford: Clarendon Press, 1974.

[23] F. Brochard, P. G. de Gennes. J. Phys. (Paris). 1970. V. 31. P. 691.

Поступила в редакцию 25.IX.1996 г.

\section{V.Yu. Novokshenov, A. G. Shagalov \\ BOUND STATES OF THE ELLIPTIC SINE-GORDON EQUATION}

We give a detailed study of finite-energy solutions to the elliptic sine-Gordon equation in the plane with point-like singularities. These bound-state solutions (in a sense of scalar field theory) with only one singularity at the origin demonstrate a target-like annular soliton pattern at large distance from the origin. An effective radius of this pattern is calculated both analytically and numerically for the case of axial symmetric solutions. Analytic study is based on the isomonodromic deformation method for the third Painlevé equation that distinguishes bound-state solutions as separatrices in a manifold of general (infinite-energy) solutions. Exact analytic estimes provide a tool for studing bound-state solutions to the non-integrable SG equation with forcing. Namely, for large intensity at the singularity we derive a critical value of forcing, admitting the existence and stability of the bound-state solutions. Applications to large area Josephson junctions and nematic liquid crystals in a rotating magnetic field are considered. For both examples we compute critical values of the field and driving forces enabling the formation of modes with finite energy. The results are in good correspondence with computer simulations and experimental data. 